\title{
Solar Wind Electron Acceleration via Langmuir Turbulence
}

\author{
Peter H. Yoon ${ }^{1,2, *}$, Luiz F. Ziebell ${ }^{3}$, Rudi Gaelzer ${ }^{4}$, Linghua Wang ${ }^{5}$, and Robert P. Lin ${ }^{2,5}$ \\ ${ }^{I}$ Institute for Physical Science \& Technology, University of Maryland, College Park, USA \\ ${ }^{2}$ School of Space Research, Kyung Hee University, Yongin, Korea \\ ${ }^{3}$ Instituto de Física, Universidade Federal do Rio Grande do Sul, Porto Alegre, Brazil \\ ${ }^{4}$ Instituto de Física e Matemática, Universidade do Federal Pelotas, Brazil \\ ${ }^{5}$ Space Science Laboratory, University of California, Berkeley, USA
}

Received 8 March 2012, accepted 30 May 2012

\begin{abstract}
The solar wind electrons observed at $1 \mathrm{AU}$ are characterized by velocity distribution functions (VDF) that deviate from the Maxwellian form in a high energy regime. Such a feature is often modeled by a kappa distribution. In the present paper a self-consistent theory of quiet-time solar wind electrons that contain a power-law tail component, $f \propto v^{-\alpha}$, is discussed. These electrons are assumed to be in dynamic equilibrium with enhanced electrostatic fluctuations with peak frequency near the plasma frequency (i.e., the Langmuir turbulence). In order to verify the theoretical prediction, the solar wind electrons in the high-energy range known as the super-halo distribution detected by WIND and STEREO spacecraft are compared against the theoretical model where it was found that the theoretical power-law index is intermittent with regard to the observed range of indices, thus indicating that the turbulent equilibrium model of suprathermal solar wind electrons may be valid.
\end{abstract}

Key words: Solar wind electrons, Superhalo, Langmuir turbulence, Kappa distribution

Citation: Yoon, P. H., L. F.Ziebell, R. Gaelzer, L. Wang, and R. P. Lin, 2013: Solar wind electron acceleration via langmuir turbulence. Terr. Atmos. Ocean. Sci., 24, 175-182, doi: 10.3319/TAO.2012.05.30.01(SEC)

\section{INTRODUCTION}

Observations of charged particles in the solar wind show that the velocity distribution functions (VDF) associated with these particles feature suprathermal components (Feldman et al. 1975; Gosling et al. 1981; Armstrong et al. 1983). For a high energy regime these VDFs can be characterized by a velocity power-law tail, $f \propto v^{-\alpha}$. In the literature, these non-thermal VDFs are often modeled by the kappa distribution (Vasyliunas 1968), $f(v) \propto 1 /\left[\left(1+v^{2} / \kappa v_{T}^{2}\right)^{\kappa+1}\right]$, where $v_{T}=(2 T / m)^{1 / 2}$ is the Maxwellian thermal speed. Of course, the limit of $\kappa \rightarrow \infty$ corresponds to the Maxwellian VDF. The kappa model facilitates the interpretation of observation, but it has no physical basis. Recently, however, it was realized that the kappa VDF corresponds to the most probable state in the non-extensive (or Tsallis) thermostatistics (Tsallis 1988, 2009; Livadiotis and McComas 2009).

The non-extensive concept of space plasma VDF is an intriguing and profound idea that deserves a thorough

\footnotetext{
* Corresponding author

E-mail:yoonp@umd.edu
}

examination (Tsallis 1988, 2009; Livadiotis and McComas $2009,2010,2011)$. In the present paper, however, we do not explore this approach in any great depth. Instead, we shall discuss an alternative and perhaps inter-related concept of a quasi-stationary turbulent state. Such a state is characterized by charged particles and self-consistently generated turbulence that are in stationary dynamic equilibrium. In plasmas the nature of wave-particle interaction is long-ranged, which causes correlations and impels the stationary states to deviate from (thermal) equilibrium. Non-extensive statistical theory describes such a system. Such a connection between turbulent and non-extensive state has not been explored extensively but represents fertile ground for future research.

A turbulent quasi-stationary state is different from a Vlasov equilibrium, for in a Vlasov equilibrium the level of electromagnetic fluctuations is supposed to be infinitesimal. In contrast, a turbulent stationary state is characterized by a finite level of electromagnetic fluctuations. To discuss such a state, one must consider steady-state solutions for particle and wave kinetic equations in weak turbulence theory. 
Yoon et al. $(2005,2006)$ and Rhee et al. (2006) were the first to solve the self-consistent equations of weak turbulence theory by numerical means and demonstrate that kappa-like VDF associated with the electrons may form in the time asymptotic limit, as a result of self-consistent interaction between the electrons and Langmuir turbulence. Their work thus implied that the kappa distribution of solar wind electrons might indeed correspond to the turbulent quasiequilibrium. These works are, however, based on a numerical initial value problem such that it is not evident whether the kappa-like final state obtained by numerical means does truly correspond to the asymptotic state or not. Moreover, the above papers considered simplifying one-dimensional (1D) situations only.

For these reasons, the issue of whether the equations of weak turbulence theory that describes the interaction of electrons and Langmuir turbulence lend themselves to asymptotic steady-state solution in the mathematical sense, especially in higher dimensions, deserves a separate discussion. Recently, Yoon (2011, 2012a, b) carried out such an analysis, and we shall briefly outline the basic idea in a subsequent section.

In the present paper we present the essential findings related to the problem of self-consistent formation of electron kappa-like VDF by interaction with Langmuir turbulence. We shall also present observational evidence on the basis of WIND and STEREO measurement made during quiet time near $1 \mathrm{AU}$, that shows that theoretical prediction of the velocity power-law index $\alpha$ is indeed consistent with actual solar wind superhalo electron observations.

The organization of the present paper is as follows: In section 2 we present the basic set of equations of weak turbulence theory which describe the self-consistent interaction of electrons and Langmuir/ion-sound turbulence. We present an example of how Langmuir turbulence and kappalike electron VDF develop in a quasi-asymptotic regime by considering an initial value problem of an electron-beam system. In section 3 we offer an overview for the theory of asymptotic steady-state solution of the weak turbulence theory. Such a solution represents the turbulent equilibrium for the electron-Langmuir wave system. Finally, in section 4 , we present an example taken from the solar wind where we show the quiet-time solar wind electron VDF. Upon comparison with theoretical prediction of velocity space power-law index and observed values, we find that the two are not inconsistent. Finally, section 5 concludes with final thoughts.

\section{ELECTRON ACCELERATION BY LANGMUIR SPECTRUM}

The dynamics of the system of electron-Langmuir/ ion-sound turbulence is described by the equations of weak turbulence theory (Yoon et al. 2005, 2006), which first in- volves the electron particle kinetic equation,

$$
\begin{aligned}
& \frac{\partial f_{e}}{\partial t}=\frac{\partial}{\partial v_{i}}\left(A_{i} f_{e}+D_{i j} \frac{\partial f_{e}}{\partial v_{j}}\right) \\
& A_{i}=\frac{e^{2}}{4 \pi m_{e}} \int d \mathbf{k} \frac{k_{i}}{k^{2}} \sum_{\sigma= \pm 1}\left(\sigma \omega_{\mathbf{k}}^{L}\right) \delta\left(\sigma \omega_{\mathbf{k}}^{L}-\mathbf{k} \cdot \mathbf{v}\right) \\
& D_{i j}=\frac{\pi e^{2}}{m_{e}^{2}} \int d \mathbf{k} \frac{k_{i} k_{j}}{k^{2}} \sum_{\sigma= \pm 1} \delta\left(\sigma \omega_{\mathbf{k}}^{L}-\mathbf{k} \cdot \mathbf{v}\right) I_{\mathbf{k}}^{\sigma L}
\end{aligned}
$$

where $f_{e}$ is the electron VDF (normalized to unity, $\int d \mathbf{v} f_{e}=$ $1), e$ and $m_{e}$ are unit charge and electron mass, respectively, $I_{\mathrm{k}}^{\sigma L}$ represents the Langmuir turbulence spectral intensity. The familiar Langmuir wave dispersion relation is given by

$\omega_{\mathbf{k}}^{L}=\omega_{p e}\left(1+\frac{3}{2} k^{2} \lambda_{D e}^{2}\right)$

where $\omega_{p e}=\left(4 \pi n e^{2} / m_{e}\right)^{1 / 2}$ is the plasma frequency, $n$ being the ambient density and $\lambda_{D e}=T_{e} /\left(4 \pi n e^{2}\right)^{1 / 2}$ is the Debye length. The equation for Langmuir waves is given by

$$
\begin{aligned}
& \frac{\partial I_{\mathbf{k}}^{\sigma L}}{\partial t}=\frac{\pi \omega_{p e}^{2}}{k^{2}} \int d \mathbf{v}\left(\sigma \omega_{\mathbf{k}}^{L}-\mathbf{k} \cdot \mathbf{v}\right)\left(\frac{n e^{2}}{\pi} f_{e}+\sigma \omega_{\mathbf{k}}^{L} I_{\mathbf{k}}^{\sigma L} \cdot \frac{\partial f_{e}}{\partial \mathbf{v}}\right) \\
& +\frac{\pi e^{2}}{2 T_{e}^{2}} \sum_{\sigma^{\prime}, \sigma^{\prime}= \pm 1} \sigma \omega_{\mathbf{k}}^{L} \int d \mathbf{k} \frac{\mu_{\mathbf{k}-\mathbf{k}^{\prime}}\left(\mathbf{k} \cdot \mathbf{k}^{\prime}\right)}{k^{2} k^{\prime 2}\left(\mathbf{k}-\mathbf{k}^{\prime}\right)} \delta\left(\sigma \omega_{\mathbf{k}}^{L}-\sigma^{\prime} \omega_{\mathbf{k}^{\prime}}^{L}-\sigma^{\prime \prime} \omega_{\mathbf{k}-\mathbf{k}^{\prime}}^{S}\right) \\
& \times\left(\sigma \omega_{\mathbf{k}}^{L} I_{\mathbf{k}^{\prime}}^{\sigma^{\prime} L} \frac{I_{\mathbf{k}-\mathbf{k}^{\prime}}^{\sigma^{\prime} S}}{\mu_{\mathbf{k}-\mathbf{k}^{\prime}}}-\sigma^{\prime} \omega_{\mathbf{k}^{\prime}}^{L} \frac{I_{\mathbf{k}-\mathbf{k}^{\prime}}^{\sigma^{\prime} S}}{\mu_{\mathbf{k}-\mathbf{k}^{\prime}}} I_{\mathbf{k}}^{\sigma L}-\sigma^{\prime \prime} \omega_{\mathbf{k}-\mathbf{k}^{\prime}}^{L} I_{\mathbf{k}^{\prime}}^{\sigma^{\prime} L} I_{\mathbf{k}}^{\sigma L}\right) \\
& -\sigma \omega_{\mathbf{k}}^{L} \frac{\pi e^{2}}{m_{e}^{2} \omega_{p e}^{2}} \sum_{\sigma= \pm 1} \int d \mathbf{k}^{\prime} \int d \mathbf{v} \frac{\left(\mathbf{k} \cdot \mathbf{k}^{\prime}\right)^{2}}{k^{2} k^{\prime 2}} \delta\left[\sigma \omega_{\mathbf{k}}^{L}-\sigma^{\prime} \omega_{\mathbf{k}^{\prime}}^{L}-\left(\mathbf{k}-\mathbf{k}^{\prime}\right) \cdot \mathbf{v}\right] \\
& \times\left[\frac{n e^{2}}{\pi \omega_{p e}^{2}}\left(\sigma^{\prime} \omega_{\mathbf{k}^{\prime}}^{L} I_{\mathbf{k}}^{\sigma L}-\sigma \omega_{\mathbf{k}}^{L} I_{\mathbf{k}^{\prime}}^{\sigma^{\prime} L}\right) f_{i}-\frac{m_{e}}{m_{i}} I_{\mathbf{k}}^{\sigma L} I_{\mathbf{k}^{\prime}}^{\sigma^{\prime} L}\left(\mathbf{k}-\mathbf{k}^{\prime}\right) \cdot \frac{\partial f_{i}}{\partial \mathbf{v}}\right]
\end{aligned}
$$

where we have defined the quantity $\mu_{\mathrm{k}}$

$\mu_{\mathrm{k}}=|k|^{3} \lambda_{D e}^{3}\left(\frac{m_{e}}{m_{i}}\right)^{1 / 2}\left(1+\frac{3 T_{i}}{T_{e}}\right)^{1 / 2}$

The first term on the right-hand side of Eq. (3) describes the spontaneous and induced emissions. The second terms feature products of wave intensities involving Langmuir and ion-sound modes, and describe three-wave decay processes. The third term describes the scattering of Langmuir waves off thermal ions. The wave kinetic equation for ion-sound waves is given by

$\frac{\partial}{\partial t} \frac{I_{\mathbf{k}}^{\sigma S}}{\mu_{\mathbf{k}}}=\frac{\pi \mu_{\mathbf{k}} \omega_{p e}^{2}}{k^{2}} \int d \mathbf{v} \delta\left(\sigma \omega_{\mathbf{k}}^{S}-\mathbf{k} \cdot \mathbf{v}\right)\left[\frac{n e^{2}}{\pi}\left(f_{e}+f_{i}\right)+\right.$ 


$$
\begin{aligned}
& \left.\sigma \omega_{\mathbf{k}}^{L} \frac{I_{\mathbf{k}}^{\sigma S}}{\mu_{\mathbf{k}}} \mathbf{k} \cdot \frac{\partial}{\partial \mathbf{v}}\left(f_{e}+\frac{m_{e}}{m_{i}} f_{i}\right)\right]+\frac{\pi e^{2}}{4 T_{e}^{2}} \sum_{\sigma^{\prime}, \sigma^{\prime}= \pm 1} \sigma \omega_{\mathbf{k}}^{L} . \\
& \int d \mathbf{k}^{\prime} \frac{\mu_{\mathbf{k}}\left[\mathbf{k}^{\prime} \cdot\left(\mathbf{k}-\mathbf{k}^{\prime}\right)\right]^{2}}{k^{2} k^{\prime 2}\left(\mathbf{k}-\mathbf{k}^{\prime}\right)^{2}} \delta\left(\sigma \omega_{\mathbf{k}}^{S}-\sigma^{\prime} \omega_{\mathbf{k}^{\prime}}^{L}-\sigma^{\prime \prime} \omega_{\mathbf{k}-\mathbf{k}^{\prime}}^{L}\right)
\end{aligned}
$$

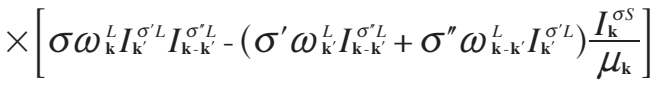

In the above $I_{\mathbf{k}}^{\sigma S}$ represents the ion-sound wave intensity and the ion-sound dispersion relation is given by

$$
\omega_{\mathrm{k}}^{S}=\omega_{p e} \frac{k \lambda_{D e}}{\left(1+k^{2} \lambda_{D e}^{2}\right)^{1 / 2}}\left(\frac{m_{e}}{m_{i}}\right)^{1 / 2}\left(1+\frac{3 T_{i}}{T_{e}}\right)^{1 / 2}
$$

The quantities $I_{\mathrm{k}}^{\sigma L}$ and $I_{\mathrm{k}}^{\sigma S}$ are defined via the total electrostatic field spectral wave energy densitites as

$$
\delta E_{\mathrm{k} \omega}^{2}=\sum_{\sigma= \pm 1}\left[I_{\mathrm{k}}^{\sigma L} \delta\left(\omega-\sigma \omega_{\mathrm{k}}^{L}\right)+I_{\mathrm{k}}^{\sigma S} \delta\left(\omega-\sigma \omega_{\mathrm{k}}^{S}\right)\right]
$$

The quantities, $\sigma, \sigma^{\prime}, \sigma^{\prime \prime}= \pm 1$ designate the direction of wave propagation, plus/minus sign representing forward/ backward direction. Other quantities such as $\omega_{\mathbf{k}^{\prime}}^{L}, \omega_{\mathbf{k}-\mathbf{k}^{\prime}}^{L}$, $\omega_{\mathbf{k}^{\prime}}^{S}, \omega_{\mathbf{k}-\mathbf{k}^{\prime}}^{S}$ simply denote Langmuir or ion-sound mode dispersion relations (2) or (6) with different wave vector arguments.

In order to demonstrate the electron acceleration via Langmuir turbulence, let us consider the initial value problem in which the initial electron VDF is given by a Maxwellian core plus a tenuous energetic beam distribution. We consider a one-dimensional (1D) situation for the sake of simplicity. We consider that the electron thermal speed associated with the core component and the beam component is the same but the beam electrons have a net drift speed that is four times that of thermal speed. We also consider a $1 \%$ beam number density. The ions are considered to be stationary. Since the dynamic time scale associated with the Langmuir wave generation is much shorter than typical ion temporal scale, it is reasonable to consider the ions to be stationary. We set the ion-to-electron temperature ratio to $1 / 7$. This number is arbitrary, but it must be, in general, small in order to avoid heavy damping of the ion-sound waves. The initial Langmuir wave and ion-sound wave intensity levels are taken at a noise level. We have solved the set of Eqs. (1) - (6) by numerical means; the result is shown in Fig. 1.

In Fig. 1 we have considered the value of the plasma parameter $g=1 /\left(n \lambda_{D e}^{3}\right)=5 \times 10^{-3}$. The plasma parameter, which is the inverse of the number of particles in a sphere of radius equal to the Debye length, is a measure of the collisionality, the smaller the value, the more collisionless the plasma.
We also have used the dimensionless wave number $q=k v_{e} / \omega_{p e}$ and dimensionless time $\tau=\omega_{p e} t$, where $v_{e}=$ $\left(2 T_{e} / m_{e}\right)^{1 / 2}$ is the thermal speed. We have integrated, numerically, the weak turbulence equation up to $\tau=20000$, although the wave intensities are shown only up to $\tau=10000$. The initial levels are shown with dark gray lines and the final wave levels are shown with red lines. The intermediate solutions are displayed with various shades of colors. At early temporal ranges, only the primary Langmuir waves around $q=0.25$ are amplified as a result of the bump-in-tail instability. However, in the nonlinear stage of the evolution, the combined effects of three-wave decay and nonlinear scattering leads to the generation of back-scattered Langmuir waves with q roughly equal to $q=-0.5$. Note also that long wavelength (or short $q$ ) Langmuir waves are generated in the nonlinear stage. These small $q$ Langmuir waves are known as condensate modes are responsible for accelerating/heating the electrons to suprathermal energies. The generation of ion-sound waves is entirely because of the decay instability.

The electron kinetic Eq. (1) is also solved together with the wave kinetic equations for Langmuir and ion-sound waves. Figure 2 displays the electron VDF as it evolves from the initial Gaussian core plus beam into a quasi-kappalike VDF. For relatively early time, the familiar quasilinear velocity space plateau formation can be seen. However, over a long time scale, a significant heating of the electrons in the suprathermal range can be seen. The heated electrons form suprathermal tail component which can be fitted with a phenomenological kappa distribution with index $\kappa=3.5$. The primary reason for the acceleration of electrons is the formation of Langmuir condensate mode. Small wave number allows electrons with high velocity to participate in the wave-particle resonance; this process leads to the formation of an energetic tail component.

We have shown in Figs. 1 and 2 that, by solving numerically the weak turbulence equation one may demonstrate the acceleration/heating of electrons by self-consistently generated Langmuir turbulence. However, such a demonstration is based upon numerical initial value problem in 1D. It is not evident whether the final kappa-like VDF is indeed the true steady-state solution or not from a mathematical standpoint. Therefore, it is of interest to obtain the true time-asymptotic steady-state turbulent equilibrium solution directly by solving the steady-state weak turbulence equation.

\section{STEADY-STATE TURBULENCE AND SUPRATH- ERMAL ELECTRONS}

In a recent series of works one of the present authors discussed the asymptotic electron VDF based upon the equations of weak turbulence theory (Yoon 2011, 2012a). Here, we briefly outline the solution. If we consider the steadystate electron kinetic equation, 

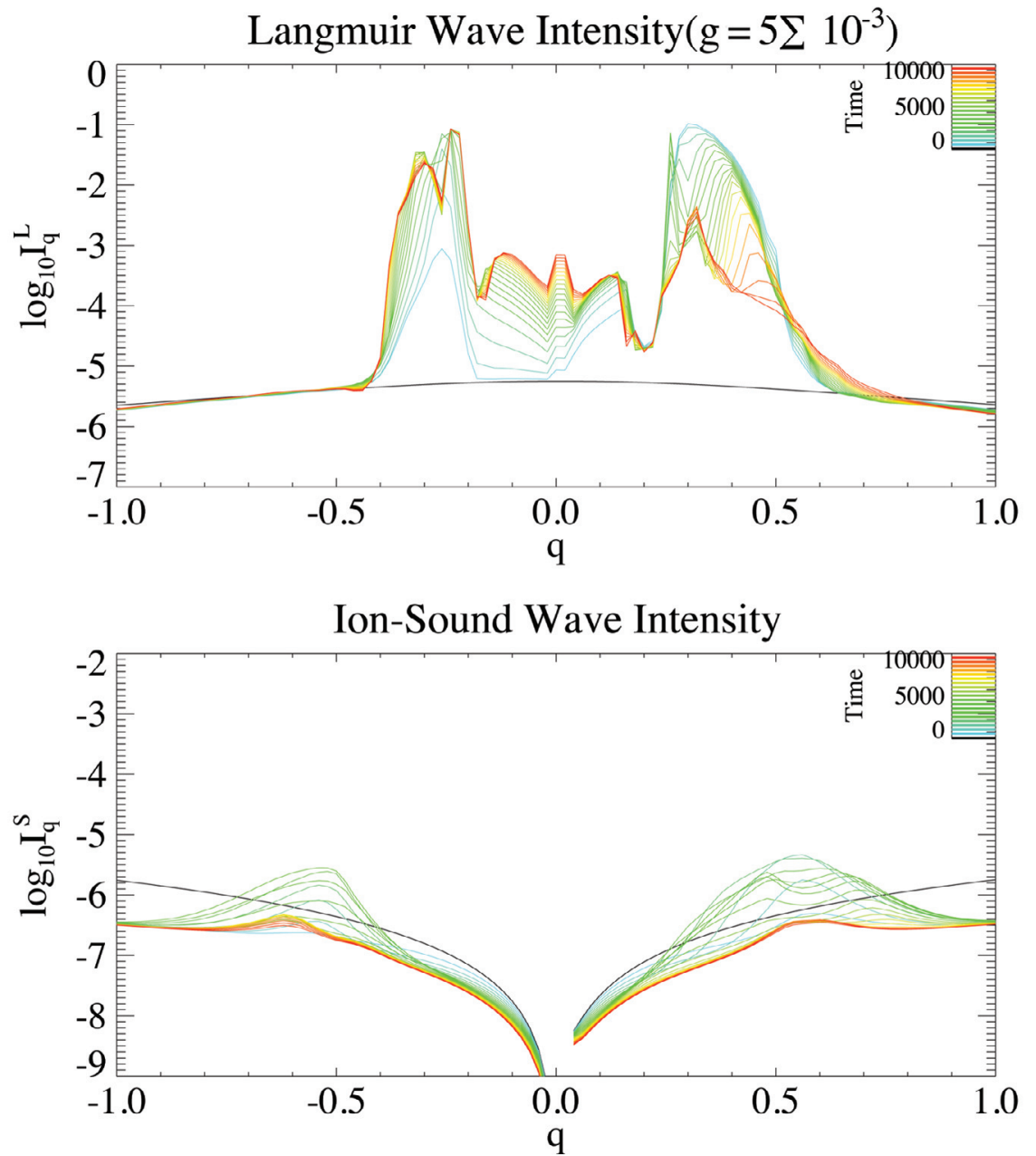

Fig. 1. Langmuir and ion-sound wave intensities versus normalized wave number and dimensionless time.

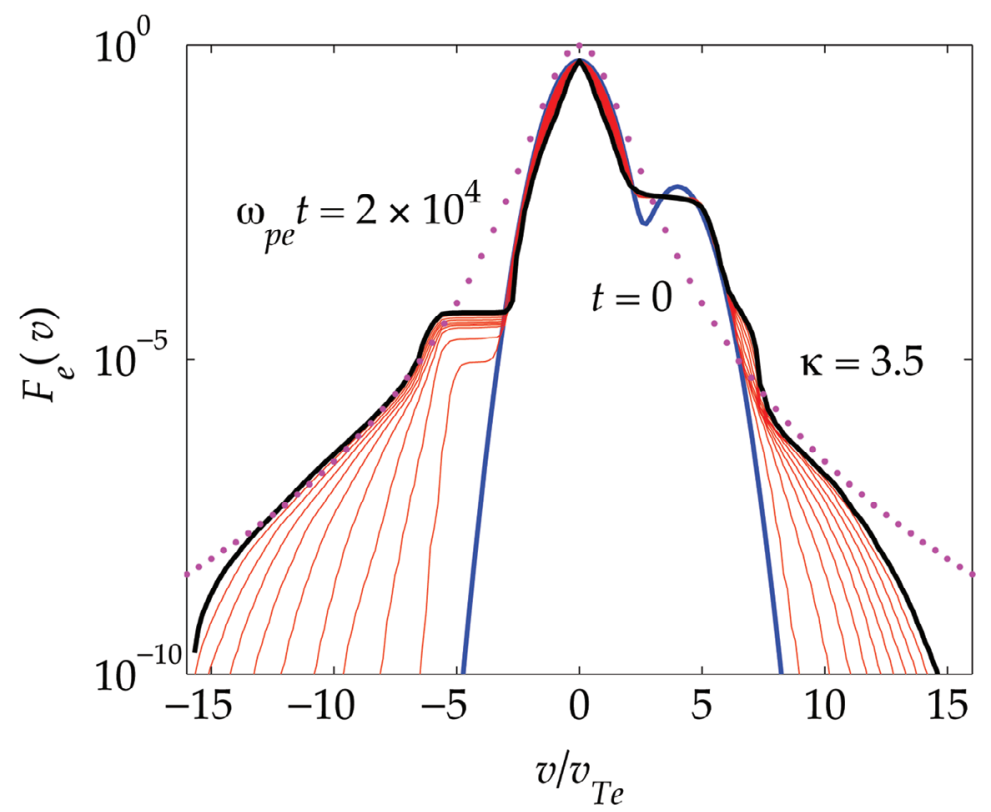

Fig. 2. Electron distribution function versus velocity, which shows evolution from the initial Gaussian core plus beam VDF to quasi-kappa VDF. Kappa value of 3.5 is used to fit the final solution (dotted lines). 
$0=\frac{\partial}{\partial v_{i}}\left(A_{i} f_{e}+D_{i j} \frac{\partial f_{e}}{\partial v_{j}}\right)$

then, by rewriting the above in spherical velocity coordinate system, and assuming that the solution is isotropic, we may show that the formal solution is given by

$f_{e}=C \exp \left(-\int d v \frac{\nu G}{D}\right)$

where

$$
\begin{aligned}
\frac{v G}{D} & =\frac{m_{e} v}{4 \pi^{2}} \frac{\sum_{\sigma= \pm 1} \int d \mathbf{k} k^{-2} \delta\left(\sigma \omega_{p e}-\mathbf{k} \cdot \mathbf{v}\right)}{\sum_{\sigma= \pm 1} \int d \mathbf{k} k^{-2} \delta\left(\sigma \omega_{p e}-\mathbf{k} \cdot \mathbf{v}\right) I(\mathbf{k})} \\
& \approx \frac{m_{e} v}{4 \pi^{2}} \frac{\int_{\omega_{p e / v}}^{\infty} d k k^{-1}}{\int_{\omega_{p e} / v}^{\infty} d k k^{-1} I(k)}
\end{aligned}
$$

In the above $I(\mathbf{k})$ represents the steady-state Langmuir wave spectrum. We have assumed that $I(\mathbf{k})$ is isotropic. Upon defining

$$
H(v)=\int_{\omega_{p e} / v}^{\infty} \frac{d k}{k}, \quad H(v) W(v)=\int_{\omega_{p e} / v}^{\infty} \frac{d k}{k} I(k)
$$

One may show that the following set of three-dimensional isotropic electron VDF and the associated three-dimensional isotropic Langmuir wave intensity are mutually consistent solutions within the context of the formal solutions (9) - (11):

$$
\begin{aligned}
& f_{e}(v)=A\left[1+\frac{v^{2}}{(\kappa-3 / 2) v_{e}^{2}}\right]^{\kappa-1} \\
& A=\frac{1}{\pi^{3 / 2} v_{e}^{3}} \frac{\Gamma(\kappa+1)}{(\kappa-3 / 2)^{3 / 2} \Gamma(\kappa-1 / 2)} \\
& W(k)=\frac{T_{e}}{4 \pi^{2}} \frac{\kappa-3 / 2}{\kappa}\left[1+\frac{\omega_{p e}^{2}}{(\kappa-3 / 2) k^{2} v_{e}^{2}}\right]
\end{aligned}
$$

In the above the parameter $\kappa$ is arbitrary. However, as we shall show later, it can be determined unambiguously upon considering the steady-state wave intensity. Since the Langmuir intensity $I(k)$ is defined through $W(k)$, one can see that Eq. (12) represents a closed solution. However, the question is whether the solution $W(k)$, or for that matter, $\mathrm{I}(\mathrm{k})$ that follows, also satisfies the steady-state wave kinetic equation for the Langmuir turbulence.
To discuss the self-consistent steady-state turbulence spectrum, we now consider the wave kinetic equation with the assumption of $\partial / \partial t=0$. Let us first ignore the nonlinear wave coupling, and also ignore ion-sound waves. As shown in Fig. 1, the ion-sound mode participates in the wave-wave interaction but in the asymptotic stage, the wave intensity associated with the ion-sound mode is reduced down to the level of initial wave level. Moreover, the ion-sound mode only participates in the wave-wave interaction, and since wave-wave processes do not exchange momentum and energy with the particles, we may ignore such processes in the discussion of asymptotic solutions. The balance of spontaneous and induced emissions in the Langmuir wave kinetic equation,

$0=\frac{\pi \omega_{p e}^{2}}{k^{2}} \int d \mathbf{v}\left(\sigma \omega_{\mathbf{k}}^{L}-\mathbf{k} \cdot \mathbf{v}\right)\left(\frac{n e^{2}}{\pi} f_{e}+\sigma \omega_{\mathbf{k}}^{L} \mathbf{K}_{\mathbf{k}}^{\sigma L} \cdot \frac{\partial f_{e}}{\partial \mathbf{v}}\right)$

leads to

0

$$
\begin{aligned}
& =\int_{0}^{\infty} d k \int d \mathbf{v}\left(\omega_{p e}-k v \mu\right)\left[1-\frac{\kappa+1}{\kappa-3 / 2} \frac{4 \pi^{2}}{T_{e}} \frac{I(k)}{1+v^{2} /(\kappa-3 / 2) v_{e}^{2}}\right] f_{e} \\
& =4 \pi \int_{0}^{\infty} d v v\left[H(v)-\frac{\kappa+1}{\kappa-3 / 2} \frac{4 \pi^{2}}{T_{e}} \frac{H(v) W(k)}{1+v^{2} /(\kappa-3 / 2) v_{e}^{2}}\right] f_{e}
\end{aligned}
$$

upon making use of the electron VDF given in Eq. (12). From Eq. (14) it readily follows that $W(k)$ given by Eq. (12) is indeed the self-consistent solution of the steady-state wave kinetic equation if we ignore the nonlinear coupling term. Here, we remind the readers again that in the solution (12), the parameter $\kappa$ is arbitrary. To determine this quantity, we now turn to nonlinear term in the wave kinetic equation. For this purpose, however, we ignore wave-wave interaction terms, for reasons discussed already.

The balance of spontaneous and induced scattering terms can be achieved by considering the nonlinear waveparticle interaction terms in the Langmuir wave kinetic Eq. (3)

$$
\begin{aligned}
& 0=\sum_{\sigma= \pm 1} \int d \mathbf{k}^{\prime} \int d \mathbf{v} \frac{\left(\mathbf{k} \cdot \mathbf{k}^{\prime}\right)^{2}}{k^{2} k^{\prime 2}} \delta\left[\sigma \omega_{\mathbf{k}}^{L}-\sigma^{\prime} \omega_{\mathbf{k}^{\prime}}^{L}-\left(\mathbf{k}-\mathbf{k}^{\prime}\right) \cdot \mathbf{v}\right] \\
& \times\left[\frac{n e^{2}}{\pi \omega_{p e}^{2}}\left(\sigma^{\prime} \omega_{\mathbf{k}^{\prime}}^{L} I_{\mathbf{k}}^{\sigma L}-\sigma \omega_{\mathbf{k}}^{L} I_{\mathbf{k}^{\prime}}^{\sigma^{\prime} L}\right) f_{i}-\frac{m_{e}}{m_{i}} I_{\mathbf{k}}^{\sigma L} I_{\mathbf{k}^{\prime}}^{\sigma^{\prime} L}\left(\mathbf{k} \cdot \mathbf{k}^{\prime}\right) \cdot \frac{\partial f_{i}}{\partial \mathbf{v}}\right]
\end{aligned}
$$

Upon simplifying the above equations by retaining only those terms which satisfy the nonlinear wave-particle resonance conditions we arrive at 
$0=\int d \mathbf{k}^{\prime} \int d \mathbf{v} \frac{\left(\mathbf{k} \cdot \mathbf{k}^{\prime}\right)^{2}}{k^{2} k^{\prime 2}} \delta\left[\omega_{\mathbf{k}}^{L}-\omega_{\mathbf{k}^{\prime}}^{L}-\left(\mathbf{k}-\mathbf{k}^{\prime}\right) \cdot \mathbf{v}\right]$

$\times\left\{\frac{T_{i}}{4 \pi^{2}}\left[\omega_{\mathbf{k}^{\prime}}^{L} I(\mathbf{k})-\omega_{\mathbf{k}}^{L} I\left(\mathbf{k}^{\prime}\right)\right]+I(\mathbf{k}) I\left(\mathbf{k}^{\prime}\right)\left(\omega_{\mathbf{k}}^{L}-\omega_{\mathbf{k}^{\prime}}^{L}\right)\right\} f_{i}$

If we write $\mathbf{k}^{\prime}=\mathbf{k}+\delta \mathbf{k}$, and assume that $\delta \mathbf{k}$ is small, then Eq. (16) can be further expressed as

$$
\begin{aligned}
& 0=\int d(\delta \mathbf{k}) \int d \mathbf{v} \frac{\left(\mathbf{k} \cdot \mathbf{k}^{\prime}\right)^{2}}{k^{2} k^{\prime 2}} \delta\left[\omega_{\mathbf{k}}^{L}-\omega_{\mathbf{k}^{\prime}}^{L}-\left(\mathbf{k}-\mathbf{k}^{\prime}\right) \cdot \mathbf{v}\right] \\
& \times \delta \mathbf{k} \cdot\left\langle\frac{d I(\mathbf{k})}{d \mathbf{k}}+\frac{d \omega_{\mathbf{k}}^{L} / d \mathbf{k}}{\omega_{\mathbf{k}}^{L}}\left\{\frac{4 \pi^{2}}{T_{i}}[I(\mathbf{k})]^{2}-I(\mathbf{k})\right\}\right\rangle f_{i}
\end{aligned}
$$

By equating the quantities within the large square bracket in the second line of Eq. (17), we may obtain the solution $I(k)$ that satisfies the nonlinear wave kinetic Eq. (17),

$$
I(k)=\frac{T_{i}}{4 \pi^{2}}\left(1+\frac{4}{3} \frac{1}{k^{2} v_{e}^{2} / \omega_{p e}^{2}}\right)
$$

If we compare the basic functional form of Eq. (18) with $W(k)$ given by Eq. (12), we may easily conclude that

$$
=9 / 4=2.25 \text { and } T_{i} / T_{e}=3 / 13
$$

An important consequence of the above findings is that for high-energy tail velocity range, the self-consistent electron VDF in Eq. (12) must behave as a velocity power-law distribution,

$f_{e}(v) \propto \frac{1}{v^{2 \kappa+2}} \approx \frac{1}{v^{6.5}}$

In order to verify whether such a prediction may satisfactorily explain the observation, we now turn to spacecraft observation of solar wind electrons near $1 \mathrm{AU}$ during quiet time.

\section{SOLAR WIND ELECTRONS}

We performed a statistical survey of $\sim 2$ - $20 \mathrm{keV}$ superhalo electron observations during quiet times in the interplanetary medium in 2007 - 2008, from the SupraThermal Electron (STE) instrument onboard the STEREO A \& B spacecraft. The observed quiet-time VDFs of superhalo electrons fit well to a power-law, $f_{e} \sim v^{\text {-b }}$, from 2 to $20 \mathrm{keV}$, with $b$ ranging from 5 to 8.7 with an average of $6.69 \pm 0.90$ and about half of the points in a peak at $6.5<b<7.5$. We chose to compare the superhalo power-law index with the theoretical prediction since the lower velocity range elec- tron population, often called the halo, has a mixture of suprathermal plus strahl components, such that it is not easy to extract a clear power-law index. In contrast, the superhalo component clearly shows well-defined power-law behavior. Figure 3 shows that on 9 January 2007 when WIND and two STEREO spacecrafts were close together $\left(<140 R_{E}\right.$ or $0.06 \mathrm{AU}$ ), the observed superhalo VDFs show similar power-laws, $f_{e} \sim v^{-7.3}$. About 10 month later when the STEREO spacecraft were $\sim 42$ deg ecliptic longitude (0.7 AU) apart, the superhalo electrons (Fig. 3, insert) show significantly different power-laws (exponents of -5.3 and -6.3 ) at the two spacecraft, indicating variations on that spatial scale, and possibly temporal variation on a scale of months. Note that the role of WIND spacecraft is to provide continuous electron VDF coverage over the halo region, but it does not directly affect the superhalo measurement.

Such a variation is not unexpected since the real solar wind is not in exact dynamical equilibrium. Nevertheless, judging from the fact that theoretical prediction of $f_{e} \sim v^{-6.5}$ is intermediate between observed range of power-law indices $\left(\sim v^{-5.0}\right.$ to $v^{-8.7}$ with average $\left.v^{-6.69}\right)$, we find that the agreement is quite remarkable.

\section{CONCLUSIONS AND DISCUSSION}

In the present paper we discussed the problem of (local) acceleration of suprathermal electrons arising from Langmuir turbulence. We presented an example of selfconsistent electron acceleration by solving numerically the equations of weak turbulence theory. In order to facilitate the discussion, we chose the problem of bump-in-tail instability so that the free energy associated with the electron beam leads to the rapid excitation of Langmuir turbulence and subsequent saturation. Of course in the quiet-time solar wind, no electron beam with a positive gradient in velocity space is observed at $1 \mathrm{AU}$. However, the solar wind contains the energetic, highly field-aligned component called the strahl. The enhanced Langmuir fluctuation excited by these strahl's may drive the system to time-asymptotic steady-state turbulence for the Langmuir wave spectrum. It should be noted, however, that we are concerned largely with the time-asymptotic state associated with the Langmuir turbulence such that the choice of initial configuration should not matter.

We have also presented the theory of steady-state Langmuir turbulence and electron acceleration problem by solving the set of coupled electron-Langmuir turbulence equations. On the basis of such an analysis, we found that the steady-state suprathermal electron should behave as $f_{e} \sim v^{-6.5}$. In order to verify this theoretical prediction against observation, we took the quiet-time solar wind electrons as a representative example of quasi-steady state particle population, and compared the velocity power-law spectral indices predicted by both theory and observation. We found 


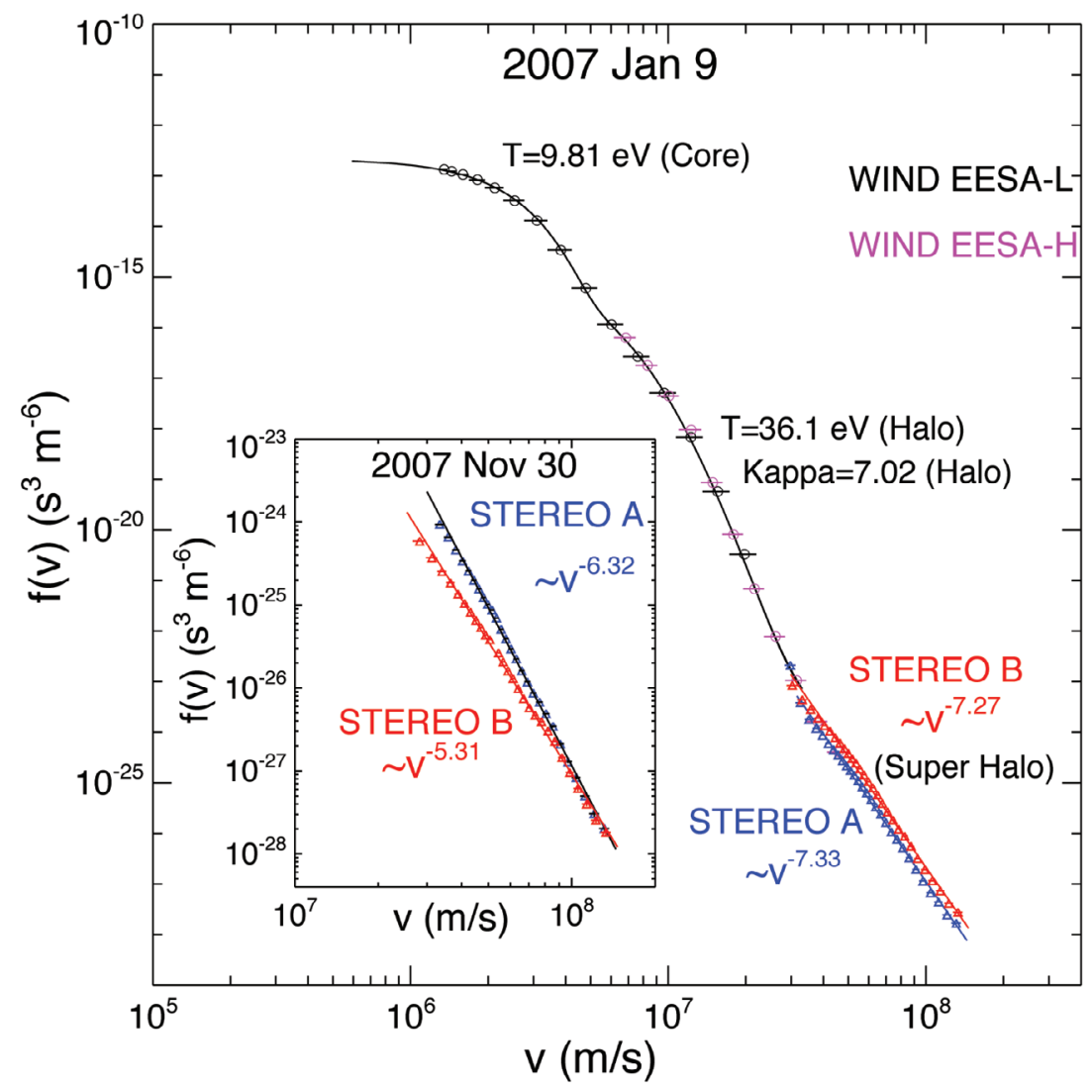

Fig. 3. Omnidirectional electron VDF measured from $\sim 10^{6} \mathrm{~m} \mathrm{~s}^{-1}(\sim 5 \mathrm{eV})$ to $\sim 10^{8} \mathrm{~m} \mathrm{~s}^{-1}(\sim 60 \mathrm{keV})$ during a quiet period in the interplanetary medium on 9 January 2007, The black line gives the Maxwellian fit to the solar wind (SW) core and Kappa fit to the SW halo, measured by the Wind spacecraft. The pink and blue lines are power-law fit to the solar wind superhalo measured by the STEREO A \& B spacecraft. The three spacecrafts are located within $\sim 140 \mathrm{R}_{\mathrm{E}}(0.06 \mathrm{AU})$ of each other, near L1, $\sim 200 \mathrm{R}_{\mathrm{E}}$ upstream of the Earth. The inset shows the superhalo electron spectra measured on 30 November 2007 by STEREO A \& B, separated by $\sim 0.7$ AU (20.6 deg ahead of, and 21.1 deg ecliptic longitude behind, the Earth, respectively).

a reasonable agreement between the theory $\left(v^{-6.5}\right)$ and observation $\left(\sim v^{-5.0}\right.$ to $v^{-8.7}$ with average $\left.v^{-6.69}\right)$. On the basis of this finding we believe that the local acceleration of energetic electrons by Langmuir turbulence may indeed take place in the solar wind, and that similar turbulent acceleration processes involving other types of wave modes and charged particles of different species may take place in other space environments.

Acknowledgments Peter H. Yoon acknowledges NSF grant AGS0940985 and NASA grant NNX09AJ81G to the University of Maryland. Peter H. Yoon also acknowledges WCU grant No. R31-10016 to Kyung Hee University from the Korean Ministry of Education, Science and Technology. Luiz F. Ziebell and Rudi Gaelzer acknowledge support from CNPq and FAPERGS. The research at Berkeley was supported in part by NASA grants NAS5-03131 for STEREO IMPACT and NNX10AQ31G for Wind 3DP.

\section{REFERENCES}

Armstrong, T. P., M. T. Paonessa, E. V. Bell II, and S. M.
Krimigis, 1983: Voyager observations of Saturnian ion and electron phase space densities. J. Geophys. Res., 88, 8893-8904, doi: 10.1029/JA088iA11p08893. [Link]

Feldman, W. C., J. R. Asbridge, S. J. Bame, M. D. Montgomery, and S. P. Gary, 1975: Solar wind electrons. J. Geophys. Res., 80, 4181-4196, doi: 10.1029/JA080i 031p04181. [Link]

Gosling, J. T., J. R. Asbridge, S. J. Bame, W. C. Feldman, R. D. Zwickl, G. Paschmann, N. Sckopke, and R. J. Hynds, 1981: Interplanetary ions during an energetic storm particle event: The distribution function from solar wind thermal energies to $1.6 \mathrm{MeV}$.J. Geophys. Res., 86, 547-554, doi: 10.1029/JA086iA02p00547. [Link]

Livadiotis, G. and D. J. McComas, 2009: Beyond kappa distributions: Exploiting Tsallis statistical mechanics in space plasmas. J. Geophys. Res., 114, A11105, doi: 10.1029/2009JA014352. [Link]

Livadiotis, G. and D. J. McComas, 2010: Exploring transitions of space plasmas out of equilibrium. Astrophys. J., 714, 971-987, doi: 10.1088/0004-637X/714/1/971. [Link] 
Livadiotis, G. and D. J. McComas, 2011: Invariant kappa distribution in space plasmas out of equilibrium. Astrophys. J., 741, 88, doi: 10.1088/0004-637X/741/2/88. [Link]

Rhee, T., C. M. Ryu, and P. H. Yoon: 2006: Self-consistent formation of electron $\kappa$ distribution: 2. Further numerical investigation. J. Geophys. Res., 111, A09107, doi: 10.1029/2006JA011682. [Link]

Tsallis, C., 1988: Possible generalization of BoltzmannGibbs statistics. J. Stat. Phys., 52, 479-487, doi: 10.10 07/BF01016429. [Link]

Tsallis, C., 2009: Introduction to Nonextensive Statistical Mechanics: Approaching a Complex World, Springer, New York, 400 pp.

Vasyliunas, V. M., 1968: A Survey of Low-Energy Electrons in the Evening Sector of the Magnetosphere with OGO 1 and OGO 3. J. Geophys. Res., 73, 2839-2884, doi: 10.1029/JA073i009p02839. [Link]
Yoon, P. H., 2011: Asymptotic equilibrium between Langmuir turbulence and suprathermal electrons. Phys. Plasmas, 18, 122303, doi: 10.1063/1.3662105. [Link]

Yoon, P. H., 2012a: Asymptotic equilibrium between Langmuir turbulence and suprathermal electrons in three dimensions. Phys. Plasmas, 19, 012304, doi: 10.1063/1.3676159. [Link]

Yoon, P. H., 2012b: Electron kappa distribution and steadystate Langmuir turbulence. Phys. Plasmas, 19, 052301, doi: 10.1063/1.4710515. [Link]

Yoon, P. H., T. Rhee, and C. M. Ryu, 2005: Self-consistent generation of superthermal electrons by beam-plasma interaction. Phys. Rev. Lett., 95, 215003, doi: 10.1103/ PhysRevLett.95.215003. [Link]

Yoon, P. H., T. Rhee, and C. M. Ryu, 2006: Self-consistent formation of electron $\kappa$ distribution: 1. Theory. J. Geophys. Res., 111, A09106, doi: 10.1029/2006JA011681. [Link] 